\title{
D-Instantons in Non-Critical Open String Theory
}

\author{
Rui Neves* $^{*}$ \\ Department of Mathematical Sciences, University of Durham, Science Laboratories, \\ South Road, Durham DH1 3LE, United Kingdom \\ R.G.M.Neves@Durham.ac.uk \\ February 5, 2018
}

\begin{abstract}
We show that the strength of the leading non-perturbative effects in non-critical string theory is of the order $e^{-O\left(1 / \beta_{s t}\right)}$. We show how this restricts the space of consistent theories. We also identify non-critical one dimensional D-instantons as dynamical objects which exchange closed string states and calculate the order of their size. PACS codes: 11.25-w, 11.25.Pm, 11.25.Hf, 11.25.Sq. Keywords: Noncritical open string, D-instantons, Non-perturbative effects.
\end{abstract}

\section{Introduction}

Over the years string theory has been establishing itself as the leading candidate to a unified description of particle physics and gravity. The latest and exciting development has been the rediscovery of string duality [1, 2]. Particularly fascinating is the connection between this symmetry and new extended objects like the D-branes, which has given strength to the idea that boundaries and dual membranes play a significant role in the understanding of the non-perturbative phase of the dynamics of strings [3, [4]. In this letter we are interested in dual membranes in non-critical open string theory. We consider Polyakov's model and work within the DDK approach to the conformally invariant

\footnotetext{
*Research supported by J.N.I.C.T's PRAXIS XXI PhD fellowship BD/2828/93-RM.
} 
Liouville theory [5, 6]. We discuss the non-critical D-instanton in the limiting case of a one dimensional target space and show that the strength of its associated leading stringy non-perturbative effects is of the order $e^{-O\left(1 / \beta_{s t}\right)}$, where $\beta_{s t}$ is the string coupling constant. This naturally mimics the result obtained in the 26 dimensional critical theory and in the matrix models [4, \#] since the weight of holes in the world-sheet only depends on the topology. However we find that not all of the non-critical theories allowed by perturbative Weyl invariance are consistent. These theories are characterised by different positive values of the renormalised Liouville cosmological constants $\lambda_{2}$ and $\mu_{2}$, respectively associated with the renormalisation counterterms in the length of the boundary and in the area of the world-sheet. We show that only those which satisfy $\lambda_{2} \geq \mu_{2}$ with $\lambda_{2}>0, \mu_{2} \geq 0$ or those where $\lambda_{2}=0, \mu_{2} \geq 0$ lead to acceptable non-perturbative effects. We also consider the D-instanton exchange of closed string states and show that the size of the D-instanton is of the order of $\sqrt{\alpha^{\prime}} / \ln \left(1 / \lambda_{2}\right)$ for small $\lambda_{2}>0, \mu_{2} \geq 0$ or of the order of $\sqrt{\alpha^{\prime}} / \ln \left(1 / \mu_{2}^{2}\right)$ for $\lambda_{2}=0$ and small $\mu_{2}>0$. Above $\alpha^{\prime}$ is related to the string tension $\mathrm{T}=1 /\left(2 \pi \alpha^{\prime}\right)$. The extension of the analysis to the $c \leq 1$ minimal boundary conformal models is briefly discussed.

\section{Non-critical D-instantons}

For a string in a non-critical target space we have to take into the picture the nonlinear dynamics of the conformally invariant path integral Liouville theory. Here the boundary conditions we may impose on the Liouville mode are constrained by quantum Weyl invariance. So while the Dirichlet boundary conditions lead to a discontinuity in the metric as the boundary is approached, the Neumann and free boundary conditions both allow a fully smooth and Weyl invariant theory [6]. For simplicity we use Neumann boundary conditions on the Liouville mode.

We start with the disc non-critical partition function with the case of the D-instanton in mind [4]. Consider the dual of an open bosonic string theory with $k$ dimensions compactified on a torus. The dual string field satisfies homogeneous Dirichlet boundary conditions on the compact dimensions $\left.Y^{i}\right|_{B}=0, i=d-k+1, \ldots, d$ and Neumann boundary conditions on the non-compact dimensions $\partial_{\tilde{n}} Y^{m}=0, m=1, \ldots, d-k$. Omiting the 
counterterms the partition function is given by

$$
Z=\beta_{s t}^{-\chi_{o}} \int \mathcal{D}_{\tilde{g}}(Y, \tilde{g}) \exp \left(-\frac{\mathrm{T}}{2} \int d^{2} \xi \sqrt{\tilde{g}} Y \cdot \tilde{\Delta} Y\right)
$$

where for the disc $\chi_{o}=1$. Integrating the matter fields and the reparametrisation ghosts we find after the DDK renormalisation

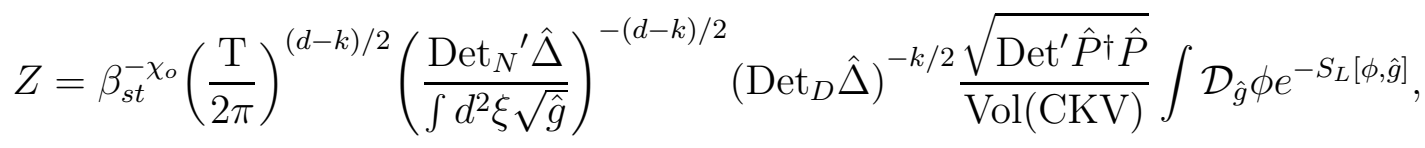

where the renormalised Liouville action is

$S_{L}[\phi, \hat{g}]=\frac{1}{8 \pi} \int d^{2} \xi \sqrt{\hat{g}}\left(\frac{1}{2} \phi \hat{\Delta} \phi+Q \hat{R} \phi\right)+\frac{Q}{4 \pi} \oint d \hat{s} k_{\hat{g}} \phi+2 \mu_{2}^{2} \int d^{2} \xi \sqrt{\hat{g}} e^{\alpha \phi}+\lambda_{2} \oint d \hat{s} e^{\alpha \phi / 2}$.

For Weyl invariance we have $Q= \pm \sqrt{(25-d) / 6}$ and for the Liouville field renormalisation $\alpha_{ \pm}=(1 / 2)\left(Q \pm \sqrt{Q^{2}-4}\right)$ [5], 6].

To integrate the Liouville mode we separate out its zero mode [8, 9]. To do so we take the semi-classical branch of $\alpha, \alpha_{-}$, which in what follows will be denoted as $\alpha$. We then write $\phi=\phi_{0}+\bar{\phi}$, where $\int d^{2} \xi \sqrt{\hat{g}} \bar{\phi}=0$. To deal with the boundary terms we have to use the Mellin transform [10]. Then the partition function is

$$
\begin{aligned}
Z= & \beta_{s t}^{-\chi_{o}}\left(\frac{\mathrm{T}}{2 \pi}\right)^{(d-k) / 2}\left(\frac{\operatorname{Det}_{N}{ }^{\prime} \hat{\Delta}}{\int d^{2} \xi \sqrt{\hat{g}}}\right)^{-(d-k) / 2}\left(\operatorname{Det}_{D} \hat{\Delta}\right)^{-k / 2} \frac{\sqrt{\operatorname{Det}^{\prime} \hat{P}^{\dagger} \hat{P}}}{\operatorname{Vol}(\mathrm{CKV})} \int \mathcal{D}_{\hat{g}} \bar{\phi} \frac{2^{s-1}}{\pi \alpha} \\
& \times\left(\int d^{2} \xi \sqrt{\hat{g}}\right)^{1 / 2}\left(2 \mu_{2}^{2} \int d^{2} \xi \sqrt{\hat{g}} e^{\alpha \bar{\phi}}\right)^{s} \Gamma(-2 s) \exp \left[\frac{\lambda_{2}^{2}\left(\oint d \hat{s} e^{\alpha \bar{\phi} / 2}\right)^{2}}{16 \mu_{2}^{2} \int d^{2} \xi \sqrt{\hat{g}} e^{\alpha \bar{\phi}}}\right] \\
& \times D_{2 s}\left[\frac{\lambda_{2} \oint d \hat{s} e^{\alpha \bar{\phi} / 2}}{2 \mu_{2}\left(\int d^{2} \xi \sqrt{\hat{g}} e^{\alpha \bar{\phi}}\right)^{1 / 2}}\right] e^{-S_{L}^{0}[\bar{\phi}, \hat{g}]},
\end{aligned}
$$

where $\alpha s=Q \chi_{o} / 2, D_{2 s}(z)$ are the parabolic cylinder functions [10] and

$$
S_{L}^{0}[\bar{\phi}, \hat{g}]=\frac{1}{8 \pi} \int d^{2} \xi \sqrt{\hat{g}}\left(\frac{1}{2} \bar{\phi} \hat{\Delta} \bar{\phi}+Q \hat{R} \bar{\phi}\right)+\frac{Q}{4 \pi} \oint d \hat{s} k_{\hat{g}} \bar{\phi}
$$

Take the case where $k=d$. Following Polchinski [4] the partition function on the disc is to be interpreted as minus the D-instanton action, which appears in the one Dinstanton amplitude $A_{1} \sim e^{Z}$. In the critical dimension this only gives the right weight 
$e^{-O\left(1 / \beta_{s t}\right)}$ because of the negative value of the renormalised Möbius volume [11]. Here we have the additional problem associated with the multiple scattering of the Liouville vertex operators. Let us try to calculate the partition function in the limiting case $d=1$. In this case $Q=2, \alpha=1$ lead to $s=1$. Then using $2^{s} e^{z^{2} / 4} D_{2 s}(z)=H_{2 s}(z)$ [10], where $H_{2 s}(z)$ is the Hermite polynomial of degree $2 s$, we get $H_{2}(z)=4 z^{2}-2$. Integrating over $\bar{\phi}$ we find on the upper half-plane

$$
\begin{aligned}
& Z=\frac{\Gamma(-2)}{2 \pi \beta_{s t}}\left(\operatorname{Det}_{D} \hat{\Delta}\right)^{-1 / 2}\left(\frac{\operatorname{Det}_{N} \hat{\Delta}}{\int d^{2} \xi \sqrt{\hat{g}}}\right)^{-1 / 2} \frac{\sqrt{\operatorname{Det}^{\prime} \hat{P}^{\dagger} \hat{P}}}{\operatorname{Vol}(\mathrm{CKV})} \varepsilon^{-6} \Lambda^{8} \\
& \times\left[\lambda_{2}^{2} \int_{-\infty}^{+\infty} d x d y(x-y)^{-2}-\frac{\mu_{2}^{2}}{2} \int_{-\infty}^{+\infty} d x d y y^{-2}\right] .
\end{aligned}
$$

Here $\varepsilon \rightarrow 0$ is a short distance cutoff which comes from the Green's functions calculated at equal points. We have also introduced the background gravity charge in the boundary at $\Lambda \rightarrow \infty$. To factor out the divergence associated with the Möbius invariance, we fix the bulk vertex operator on the center of the unit disc and the two boundary operators on 0 and $\pi / 2$. Then using the Möbius volume for an oriented theory [11] we find

$$
Z=-\left(\frac{3}{16 \pi}\right)^{3 / 2} \frac{4}{\pi \sqrt{\pi} \beta_{s t}}\left(\lambda_{2}^{2}-\mu_{2}^{2}\right) \sqrt{Q_{S}^{2}},
$$

where $Q_{S}^{2}=\left(\operatorname{Det}_{S}{ }^{\prime} \hat{\Delta}\right)^{-1} \sqrt{\operatorname{Det}_{S}{ }^{\prime} \hat{P}^{\dagger} \hat{P}}$ refer to the determinants on the Riemann sphere and now $\lambda_{2}$ and $\mu_{2}$ are taken as dimensionless constants.

Now, for any $s$ we may always absorb $\lambda_{2}^{2 s}$ into $\beta_{s t}$ and so obtain amplitudes which only depend on $\beta_{s t}$ and on the ratio $\mu_{2} / \lambda_{2}$. Within the DDK approach the one dimensional non-critical string theory can be viewed as a two dimensional critical theory defined in a consistent background given by the matter and Liouville systems [9]. So the ratio of the Liouville couplings defines different possible backgrounds for the critical string theory and so different non-critical theories. From perturbative quantum Weyl invariance alone all of them are allowed. However, since the zeta function regularisation of the determinants always leads to positive values, Eq. (7) shows that only for $\lambda_{2}>\mu_{2}$ we can find noncritical stringy non-perturbative effects of the order $e^{-O\left(1 / \beta_{s t}\right)}$ which are in agreement with the result found in the critical string theory and in the matrix models [4, 团. If $\lambda_{2}=\mu_{2}$ we find no non-perturbative effects. If $\lambda_{2}<\mu_{2}$ the theory is inconsistent. 
Note that Eq. (7) is only valid if $\lambda_{2}>0, \mu_{2} \geq 0$. If we take $\lambda_{2}=0, \mu_{2} \geq 0$ we just have to go back to the integral of the Liouville zero mode $\phi_{0}$ to see that in this case it does not bring an extra minus sign to $Z$. So, for $\lambda_{2}=0, \mu_{2} \geq 0$ we also find the right weight $e^{-O\left(1 / \beta_{s t}\right)}$ due to the negative value of the renormalised Möbius volume.

We may also analyse the range $d<1$. This corresponds to the coupling to 2D quantum gravity of the $c<1$ minimal boundary conformal field theories [6, 12, 13]. In this case $d=c=1-6(\beta-1 / \beta)^{2}$. Since the background gravity charge only depends on the worldsheet topology we still find $\alpha s=Q \chi_{o} / 2$, where $Q=\beta+1 / \beta$ and $\alpha=\alpha_{+}=\beta$. Then on the disc we find $s=\left(1+\beta^{2}\right) /\left(2 \beta^{2}\right)$ which is a rational number because $\beta^{2}=\left(2+k^{\prime}\right) /(2+k)$, where $k, k^{\prime}$ are positive integers. To see if the analysis of the limiting case $d=1$ still holds for general $k, k^{\prime}$ we would have to get involved with the intricate dynamics of the multiple Liouville scattering where $s$ does not have to be an integer [8, 9]. However if the boundary dominates the bulk, $\lambda_{2}>\mu_{2}$, the stringy non-perturbative effects should still be of the order $e^{-O\left(1 / \beta_{s t}\right)}$. If just one of the Liouville couplings is zero the same result is to be expected. These remarks are based on a possible Coulomb gas representation of the minimal boundary conformal field theories [6, 13]. They suggest that the strength of the non-perturbative effects is of the order $e^{-O\left(1 / \beta_{s t}\right)}$. A precise definition of the effects needs a careful analysis of the partition function on this type of models. This is beyond the scope of this work and we hope to discuss the matter in a future paper.

\section{The size of non-critical D-instantons}

To look for D-branes we may also consider the case where there is an exchange of closed string states while they are separated by a distance $\mathcal{Y}^{i} \mathcal{Y}_{i}$ [2]. The diagram for such an exchange is an open string annulus. Consider $p+1$ uncompactified dimensions $m=$ $0, \ldots, p$ and $d-p-1$ compact ones. According to T-duality we use Neumann boundary conditions for the $p+1$ D-brane coordinates and Dirichlet boundary conditions on the other compact coordinates. We consider that $\left.Y^{i}\right|_{B_{1}}=-\mathcal{Y}^{i} / 2$ and $\left.Y^{i}\right|_{B_{2}}=\mathcal{Y}^{i} / 2$, where $\mathcal{Y}^{i} \geq 0$, for all $i=p+1, \ldots, d$.

As usual the partition function is $Z=Z_{n c} Z_{c}$, where $Z_{n c}, Z_{c}$ are respectively the partition function on the non-compact and compact space. To integrate on the compact 
coordinates we have to factorise the classical solution $Y^{i}=Y_{c}^{i}+\bar{Y}^{i}$, where $\bar{Y}^{i}$ satisfies homogeneous Dirichlet conditions on both boundaries and $\tilde{\Delta} Y_{c}^{i}=0,\left.Y_{c}^{i}\right|_{B_{1}}=-\mathcal{Y}^{i} / 2$, $\left.Y_{c}^{i}\right|_{B_{2}}=\mathcal{Y}^{i} / 2$.

Then integrating the matter and the reparametrisation ghosts fields we find

$$
\begin{aligned}
& Z=V_{p+1}\left(\frac{\mathrm{T}}{2 \pi}\right)^{(p+1) / 2} \int d \tau \frac{\operatorname{det}(\psi, B)}{\sqrt{\operatorname{det}(\hat{B, B)}}} \frac{\sqrt{\operatorname{Det}^{\prime} \hat{P}^{\dagger} \hat{P}}}{\operatorname{Vol}(\mathrm{CKV})}\left(\frac{\operatorname{Det}_{N} \hat{\Delta}^{\hat{\Delta}}}{\int d^{2} \xi \sqrt{\hat{g}}}\right)^{-(p+1) / 2} \\
& \times\left(\operatorname{Det}_{D} \hat{\Delta}\right)^{-(d-p-1) / 2} \exp \left(-\frac{\mathrm{T}}{2} \int d^{2} \xi \sqrt{\hat{g}} \hat{g}^{a b} \partial_{a} Y_{c}^{i} \partial_{b} Y_{c i}\right) \int \mathcal{D}_{\tilde{g}} \varphi e^{-S_{L}[\varphi, \hat{g}]},
\end{aligned}
$$

where $V_{p+1}$ is the D-brane world volume and $\tau$ is the modulus of the annulus. In the case of the annulus $\chi_{o}=0$. So we can choose $\hat{R}=k_{\hat{g}}=0$ and there is no background gravity charge. This means that we do not have to deal with the Liouville amplitudes because $s=0$. After the DDK renormalisation we can integrate $\phi$ with Neumann boundary conditions to obtain

$$
\begin{gathered}
Z=\frac{V_{p+1} \Gamma(0)}{2 \pi \alpha}\left(\frac{\mathrm{T}}{2 \pi}\right)^{(p+1) / 2} \int d \tau \frac{\operatorname{det}(\psi, B)}{\sqrt{\operatorname{det}(B, B)}} \frac{\sqrt{\operatorname{Det}^{\prime} \hat{P}^{\dagger} \hat{P}}}{\operatorname{Vol}(\mathrm{CKV})}\left(\frac{\operatorname{Det}_{N}^{\prime} \hat{\Delta}}{\int d^{2} \xi \sqrt{\hat{g}}}\right)^{-(p+2) / 2} \\
\times\left(\operatorname{Det}_{D} \hat{\Delta}\right)^{-(d-p-1) / 2} \exp \left(-\frac{\mathrm{T}}{2} \int d^{2} \xi \sqrt{\hat{g}} \hat{g}^{a b} \partial_{a} Y_{c}^{i} \partial_{b} Y_{c i}\right)
\end{gathered}
$$

In this formula it is clear that the only effect of the Liouville mode within the DDK approach is to introduce just another dimension into the problem and so the calculation can be immediately carried out by following the steps already taken in the critical dimension [2, 14]. To describe the annulus we will choose the parameter domain to be a square $\left\{0 \leq \xi^{a} \leq 1\right\}$ of area $\tau$. Since we consider $\xi^{1}$ as the periodic coordinate we have $Y_{c}^{i}=\mathcal{Y}^{i} \xi^{2}-\mathcal{Y}^{i} / 2$. We obtain

$$
Z=V_{p+1} \Gamma(0)\left(2 \pi \alpha^{2} \mathrm{~T}\right)^{-1 / 2} \int \frac{d \tau}{\tau}\left(\frac{\mathrm{T}}{4 \pi \tau}\right)^{(p+2) / 2} \exp \left(-\mathrm{TY}^{2} \tau\right)|\eta(i \tau)|^{1-d}
$$

where $\eta$ is the Dedekind $\eta$ function.

From the asymptotics $\tau \rightarrow+\infty$ we use $|\eta(i / \tau)|=\sqrt{\tau}|\eta(i \tau)|$ to get the asymptotics for $\tau \rightarrow 0$ which are dominated by the lightest closed string states. We then get

$$
\begin{gathered}
Z=V_{p+1} \Gamma(0)\left(2 \pi \alpha^{2} \mathrm{~T}\right)^{-1 / 2} \int \frac{d \tau}{\tau}\left(\frac{\mathrm{T}}{4 \pi \tau}\right)^{(p+2) / 2} \exp \left(-\mathrm{TY}^{2} \tau\right) \tau^{(d-1) / 2}\left[e^{(d-1) \pi /(12 \tau)}\right. \\
\left.+(d-1) e^{(d-25) \pi /(12 \tau)}+\cdots\right]
\end{gathered}
$$


So we find a tower of massive poles for $d<1$ and one massless tachyon pole for $d=1$. This massless tachyon pole corresponds to the massless tachyon in the two dimensional critical theory where the Liouville mode is interpreted as Euclidean time [9]. If we consider a Coulomb gas representation of the $c \leq 1$ minimal boundary conformal field theories it is clear that we get exactly the same result as for the string because $s=0$. However this only confirms the suggestion that the order of magnitude of the non-perturbative effects should be the same as that of the string. For $p=-1$ the massless pole contribution is

$$
Z_{p}=\frac{\Gamma(0)}{2 \pi \sqrt{2}} \int d \tau \tau^{-3 / 2} \exp \left(-\mathrm{TY}^{2} \tau\right)
$$

This can be integrated using the Gamma function giving

$$
Z_{p}=-\frac{\Gamma(0)}{\sqrt{2 \pi}} \mathrm{T}^{1 / 2} \mathcal{Y}
$$

Let us now regularise the divergence associated with the above Gamma function [9]. If $\lambda_{2}>0$ we use the KPZ scaling on the disc for fixed boundary length $L$ [6] and write

$$
Z_{p}=-\ln \left(1 / \lambda_{2}\right)\left(\frac{\mathrm{T}}{2 \pi}\right)^{1 / 2} \mathcal{Y}
$$

which diverges like $\ln \left(1 / \lambda_{2}\right)$ as the now dimensionless $\lambda_{2} \rightarrow 0$. If $\lambda_{2}=0$ we use the KPZ scaling with fixed area $A$ [6] to get a similar divergence with a dimensionless $\mu_{2}^{2}$. These are the divergences which correspond to that already known to appear in the matrix models 15, 16]. Note that $\ln \left(1 / \lambda_{2}\right), \ln \left(1 / \mu_{2}^{2}\right)$ with dimensionful $\lambda_{2}, \mu_{2}$ define the Liouville wall [9].

Now recall that according to Polchinski's string picture [4] the one D-instanton amplitude is given by $A_{1} \sim e^{Z}$, where $Z$ is minus the D-instanton action. From the field theory of instantons in one dimension [17] the one instanton amplitude in the semi-classical approximation to one loop is

$$
A_{1}=A\left(\frac{\omega}{\pi \hbar}\right)^{1 / 2} e^{-S_{0} / \hbar-\omega T / 2}[1+O(\hbar)]
$$

where $S_{0}=\int_{-a}^{a} d x(2 V)^{1 / 2}$ is the instanton action, $\omega=V^{\prime \prime}( \pm a)$ and $A$ is a normalisation constant. Here $V$ is a symmetric double well potencial with two minima at $x= \pm a$, where $V( \pm a)=0$. In this theory $A_{1}$ is the one instanton contribution to the transition 
amplitude, $<a\left|e^{-H T / \hbar}\right|-a>$, for a first quantised particle of unit mass to go from $x=-a$ to $x=a$ as the Euclidean time $t$ goes from $t=-T / 2$ to $t=T / 2$ for large $T$. We consider the instanton with its center fixed at $t=0$. In this picture the instanton is a well localised extended object with a size of the order $1 / \omega$. To connect with the string description we have to interpret our dual string coordinate field $Y$ as the Euclidean time $t$. Then the string has its end points fixed in the center of the instanton. To the disc level the string can only see a point-like object being sensitive just to the instanton action. So to tree level both descriptions agree if $Z=-S_{0} / \hbar$.

In the case of two D-instantons we have seen that at low energy corresponding to a large distance $\mathcal{Y}$, they interact by exchanging the massless Klein-Gordon fields. Following Polchinski [4] the two D-instanton amplitude for point-like events is then

$$
A_{2} \sim e^{2 Z+Z_{p}}
$$

Once more from the theory of instantons [17] the two instanton amplitude in the semiclassical approximation to one loop is

$$
A_{2}=A^{2}\left(\frac{\omega}{\pi \hbar}\right)^{1 / 2} e^{-S_{0} / \hbar-\omega T / 2}[1+O(\hbar)]
$$

In the above formula $A_{2}$ is the two instanton contribution to the transition amplitude, $<-a\left|e^{-H T / \hbar}\right|-a>$, for the particle to go from $x=-a$ to $x=a$ and back to $x=-a$ as time goes from $t=-T / 2$ to $t=T / 2, T \rightarrow+\infty$. In this case the two instantons are in fact an instanton and an anti-instanton with widely separated centers respectively fixed close to $t=-T / 2$ and to $t=T / 2$. In the string picture the instantons are seen as point-like objects located at $t=-\mathcal{Y} / 2$ and at $t=\mathcal{Y} / 2$. They interact with a force $Z_{p}=-\omega \mathcal{Y} / 2$. So to one loop both descriptions agree provided

$$
\omega=\frac{\ln \left(1 / \lambda_{2}\right)}{\pi \sqrt{\alpha^{\prime}}}, \quad \lambda_{2}>0, \mu_{2} \geq 0
$$

or

$$
\omega=\frac{\ln \left(1 / \mu_{2}^{2}\right)}{\pi \sqrt{\alpha^{\prime}}}, \quad \lambda_{2}=0, \mu_{2}>0 .
$$


From the field theory semi-classical calculation of $A_{1}$ to one loop $1 / \omega$ is interpreted as the average size of the instanton. Thus we conclude that the size of the D-instantons is of the order of $\sqrt{\alpha^{\prime}} / \ln \left(1 / \lambda_{2}\right)$ for $\lambda_{2}>0, \mu_{2} \geq 0$ or of the order of $\sqrt{\alpha^{\prime}} / \ln \left(1 / \mu_{2}^{2}\right)$ for $\lambda_{2}=0$, $\mu_{2}>0$.

For the extension to the minimal boundary conformal models we consider the case $d=1, p=0$ where the scalar field is compactified on a circle of radius $R$. In this case the field is not single valued when the string is winding around the compact dimension $Y\left(\xi^{1}, \xi^{2}\right)=Y\left(\xi^{1}, \xi^{2}\right)+2 \pi n R, n=1,2, \ldots$. Thus the periodicity in $\xi^{1}$ can be written as $Y\left(\xi^{1}+1, \xi^{2}\right)=Y\left(\xi^{1}, \xi^{2}\right)+2 \pi n R$, which leads us to $Y\left(\xi^{1}, \xi^{2}\right)=2 \pi n R \xi^{1}+\bar{Y}\left(\xi^{1}, \xi^{2}\right)$, where $\bar{Y}$ is now a single valued field which in this case satisfies Neumann boundary conditions. For $\lambda_{2}>0, \mu_{2} \geq 0$ the partition function is

$$
Z=\ln \left(1 / \lambda_{2}\right) \frac{\sqrt{\alpha^{\prime}}}{12 R} .
$$

This result is to be compared with the partition function on closed surfaces which agrees with the corresponding matrix model [15, [16]. The extra factor of 2 comes from the open string Liouville zero mode integral. Also we have naturally lost the self-dual nature of the closed string. As for the closed surfaces we expect an agreement with the boundary matrix model. A proof of this however needs the explicit calculation which is beyond the scope of this work.

For the partition functions with fixed area $A$ and fixed boundary length $L$ only the integration of the Liouville zero mode is changed. For $Z(A), Z(L)$ we set $\lambda_{2}=0, \mu_{2}=0$ which are the cases where we can continue to use the DDK scaling argument [6]. Then

$$
Z(A)=\frac{1}{6 \alpha A R}, \quad Z(L)=\frac{1}{3 \alpha L R} .
$$

If as in the case of closed surfaces [16, [1] it could be proved that the partition functions of the boundary conformal models can be written as a linear combination of the partition functions of a scalar field compactified on some particular radii, it would be an easy matter to write results for these models. Work is in progress in this direction which we hope to present in a future paper.

If we consider a string which winds around the compact dimension but still has its end points located on two D-branes a distance $\mathcal{Y}$ apart we might be able to discuss 
non-perturbative phenomena in the boundary conformal models. We then have to take $Y\left(\xi^{1}, \xi^{2}\right)=2 \pi n R \xi^{1}+\mathcal{Y} \xi^{2}-\mathcal{Y} / 2+\bar{Y}\left(\xi^{1}, \xi^{2}\right)$. Thus the partition function is

$$
Z=-\ln \left(1 / \lambda_{2}\right) \frac{\sqrt{\alpha^{\prime}}}{2 \pi R} \ln \left(1-e^{-R \mathcal{Y} / \alpha^{\prime}}\right)
$$

As in the Neumann case we also find

$$
Z(A)=-\frac{1}{\pi \alpha A R} \ln \left(1-e^{-R \mathcal{Y} / \alpha^{\prime}}\right), \quad Z(L)=-\frac{2}{\pi \alpha L R} \ln \left(1-e^{-R \mathcal{Y} / \alpha^{\prime}}\right) .
$$

For $\mathcal{Y}=0$ we have an extra ultraviolet divergence due to the winding of the string around the compact dimension.

\section{Acknowledgments}

We would like to thank Paul Mansfield for valuable discussions.

\section{References}

[1] J. Polchinski, Rev. Mod. Phys. 68 (1996) 1245.

[2] J. Polchinski, ITP preprint NSF-ITP-96-145, hep-th/9611050 (1996).

[3] J. Dai, R.G. Leigh and J. Polchinski, Mod. Phys. Lett. A 4 (1989) 2073; R.G. Leigh, Mod. Phys. Lett. A 4 (1989) 2767.

[4] J. Polchinski, Phys. Rev. D 50 (1994) R6041.

[5] F. David, Mod. Phys. Lett. A 3 (1988) 1651; J. Distler and H. Kawai, Nucl. Phys. B 321 (1989) 509.

[6] P. Mansfield and R. Neves, Nucl. Phys. B 479 (1996) 82.

[7] S. Shenker, in: Random Surfaces and Quantum Gravity, eds. O. Alvarez, E. Marinari and P. Windey (Plenum, New York, 1991) p. 191.

[8] K. Aoki and E. D'Hoker, Mod. Phys. Lett. A 3 (1992) 235; Nucl. Phys. B 490 (1997) 40; Int. J. Mod. Phys. A 12 (1997) 1253. 
[9] P. Di Francesco and D. Kutasov, Nucl. Phys. B 375 (1992) 119.

[10] A. Erdélyi, W. Magnus, F. Oberhettinger and F.G. Tricomi, Bateman Manuscript Project, Higher transcendental functions, Vols. 1 and 2 (McGraw-Hill, New York, 1953); Tables of integral transforms, Vol. 1 (McGraw-Hill, New York, 1954).

[11] J. Liu and J. Polchinski, Phys. Lett. B 203 (1988) 39.

[12] J.L. Cardy, Nucl. Phys. B 240 (1984) 514; Nucl. Phys. B 324 (1989) 581; J.L. Cardy and D.C. Lewellen, Phys. Lett. B 259 (1991) 274.

[13] J. Schulze, Nucl. Phys. B 489 (1997) 580.

[14] A. Cohen, G. Moore, P. Nelson and J. Polchinski, Nucl. Phys. B 281 (1987) 127.

[15] D. Gross and I. Klebanov, Nucl. Phys. B 344 (1990) 475; Nucl. Phys. B 354 (1991) 459.

[16] M. Bershadsky and I. Klebanov, Phys. Rev. Lett. 65 (1990) 3088.

[17] S. Coleman, Aspects of symmetry (Cambridge University Press, Cambridge, 1985).

[18] P. Di Francesco, H. Saleur and J. Zuber, J. Stat. Phys. 49 (1987) 57; V. Pasquier, J. Phys. A 20 (1987) L1229. 